\title{
Is hypnotherapy helpful for irritable bowel syndrome in primary and secondary care?
}

Gut-directed hypnotherapy has been investigated extensively over the past three decades as an intervention for irritable bowel syndrome (IBS) in adults. Almost 30 studies of clinical outcomes, including ten randomised controlled trials, have been published on the topic, ${ }^{1}$ with most showing that hypnotherapy significantly improved bowel symptoms and, often, the associated quality of life and emotional symptoms. On the basis of this body of research, multiple recent reviews $^{1-4}$ have concluded that hypnotherapy is an effective treatment for IBS.

However, an important limitation of the work to date is that most studies were done in tertiary-care centres, and many mainly or exclusively included patients who were unresponsive to conventional medical treatments for IBS. Therefore, the effectiveness of hypnotherapy for IBS in primary-care or secondary-care settings, where most patients with IBS are treated, is unclear.

In The Lancet Gastroenterology \& Hepatology, Carla Flik and colleagues ${ }^{5}$ present the results of a large, randomised controlled trial that aimed to answer that question. With a sample size more than three times larger than any previous controlled trial of IBS hypnotherapy, and participation by clinicians in 11 different hospitals in the Netherlands, the trial was of unprecedented scope in this research domain.

The investigators randomly assigned 354 patients with IBS in primary and secondary care to one of three groups: individual hypnotherapy, group hypnotherapy, or an educational supportive control intervention carried out in groups. Participants in all three treatment arms received a total of six sessions, delivered every 2 weeks, of their assigned intervention.

The outcome of the trial was positive with regard to the primary endpoint, adequate relief of IBS symptoms. Patients in the two hypnotherapy treatment groups reported adequate relief at significantly higher rates than did those in the control group, immediately after the intervention and when reassessed 9 months later. That outcome would generally warrant the conclusion that hypnotherapy is helpful for treating patients with IBS in primary and secondary care. However, that conclusion seems doubtful in this instance, because none of the secondary outcome measures-which were well chosen and should have informed us of the exact benefits the patients derived from the interventionshowed any advantage from hypnosis treatment Bowel symptom severity, quality of life, psychological symptoms, health-care costs, and work disability showed no greater improvement in the hypnotherapy groups than in the control group. It is therefore hard to see that the health problem of IBS or its associated effects on emotional wellbeing and quality of life were specifically improved by hypnotherapy.

A second reason to question the efficacy of the therapy tested in this trial is that, even if adequate relief of symptoms is accepted as treatment success in the absence of any other evidence of therapeutic gains, the responder rate was unusually low: $40.8 \%$ in the individual therapy group and $33.2 \%$ in the group hypnotherapy group immediately after treatment. These success rates are at the bottom of the range compared with other studies ${ }^{1}$ on IBS hypnotherapy.

The reasons for the modest therapeutic effect in this study are uncertain, but there are a number of possible contributing factors. First, the hypnotherapy tested was a structured approach based on the Manchester group's clinical protocol in the UK, ${ }^{6}$ which has been successfully applied with high $(\geq 70 \%)$ success rates in several published IBS studies. ${ }^{7-10}$ However, the trial deviated notably from the Manchester approach by providing patients with only six therapy sessions instead of the Manchester group's typical 12-session course. Second, as the authors note in their discussion, the therapy was delivered by psychologists who were not experienced in treating IBS, and IBS-related education was not provided to the patients receiving hypnosis, even though doing so is typical in the Manchester hypnotherapy approach. Thus, the hypnotherapy tested in this study might have been suboptimal in amount or implementation. However, as the authors note, the smaller therapeutic effect in this trial compared with most hypnotherapy trials in tertiary care might have been because IBS in primary and secondary care is different to that in tertiary care-perhaps simpler in nature and with less involvement of psychological factors. 
Therefore, despite this impressive investigative effort by Flik and colleagues, it remains unclear whether gutdirected hypnotherapy is well suited for the treatment of patients with IBS in primary and secondary care, and future trials are needed to provide definitive answers.

\section{Olafur S Palsson}

Division of Gastroenterology and Hepatology, University of North Carolina at Chapel Hill, Chapel Hill, NC 27515, USA opalsson@med.unc.edu

OSP is a scientific advisory board member and stock holder of MetaMe Health, and was a co-investigator with Peter Whorwell, one of the Article authors, on a large global epidemiology study of functional gastrointestinal disorders, along with about 40 other co-investigators. That study was unrelated to the topic of this Comment

1 Palsson OS. Hypnosis treatment of gastrointestinal disorders: a comprehensive review of the empirical evidence. Am J Clin Hypn 2015; 58: 134-58.

2 Ford AC, Quigley EM, Lacy BE, et al. Effect of antidepressants and psychological therapies, including hypnotherapy, in irritable bowel syndrome: systematic review and meta-analysis. Am J Gastroenterol 2014 109: $1350-65$.
3 Laird KT, Tanner-Smith EE, Russell AC, Hollon SD, Walker LS. Comparative efficacy of psychological therapies for improving mental health and daily functioning in irritable bowel syndrome: a systematic review and meta-analysis. Clin Psychol Rev 2017; 51: 142-52.

4 Peters SL, Muir JG, Gibson PR. Review article: gut-directed hypnotherapy in the management of irritable bowel syndrome and inflammatory bowel disease. Aliment Pharmacol Ther 2015; 41: 1104-15.

5 Flik CE, Laan W, Zuithoff NPA, et al. Efficacy of individual and group hypnotherapy in irritable bowel syndrome (IMAGINE): a multicentre randomised controlled trial. Lancet Gastroenterol Hepatol 2018; published online Nov 22. http://dx.doi.org/10.1016/S2468-1253(18)30310-8.

6 Gonsakorale WM. Gut-directed hypnotherapy: the Manchester approach for treatment of irritable bowel syndrome. Int J Clin Exp Hypn 2006; 54: 27-50.

7 Miller V, Carruthers HR, Morris J, Hasan SS, Archbold S, Whorwell PJ. Hypnotherapy for irritable bowel syndrome: an audit of one thousand adult patients. Aliment Pharmacol Ther 2015; 41: 844-55.

8 Gonsalkorale WM, Houghton LA, Whorwell PJ. Hypnotherapy in irritable bowel syndrome: a large-scale audit of a clinical service with examination of factors influencing responsiveness. Am J Gastroenterol 2002; 97: 954-61.

9 Whorwell PJ, Prior A, Colgan SM. Hypnotherapy in severe irritable bowel syndrome: further experience. Gut 1987; 28: 423-25.

10 Whorwell PJ, Prior A, Faragher EB. Controlled trial of hypnotherapy in the treatment of severe refractory irritable-bowel syndrome. Lancet 1984; 2: $1232-34$ 